\section{Hitting cancer's sweet spot}

\section{By Lev Osherovich, Senior Writer}

Two reports published back to back in Nature provide the missing piece in a long-standing mystery about why cancers use glycolysis, rather than respiration, to fuel growth. ${ }^{1,2}$ The findings provide a mechanistic explanation for this metabolic process, called the Warburg effect after its discoverer, Nobel Prize-winning chemist Otto Warburg.

Warburg and others were convinced that glycolysis under aerobic conditions may be at the root of cancer, ${ }^{3}$ but the idea fell out of fashion several decades ago with the discovery of oncogenes. The new findings, together with other recent studies, ${ }^{4,5}$ bring aerobic glycolysis back into focus for cancer therapeutics.

Glycolysis is an attractive process to target because it involves wellcharacterized enzymes with essential functions that tumors are more dependent on than normal cells.

In the presence of oxygen, normal cells extract as much energy as possible from glucose primarily through mitochondrial oxidative phosphorylation and to a lesser extent from glycolysis. In contrast, the majority of solid tumors grow mostly through glycolysis, a less efficient but faster process that involves converting glucose to lactate-much like overworked muscles do. As a result, tumor cells use less oxygen and more sugar than normal cells.

Which glycolysis component to hit, however, remains an open question (see Figure 1, "Steps in glycolysis and respiration targeted by cancer therapeutics").

In a series of biochemical, cell culture and mouse experiments, the studies identified an alternative form of pyruvate kinase, called pyruvate kinase isoform M2 (PKM2), as the lynchpin of cancer-specific aerobic glycolysis.

Both reports are from a research group led by Lewis Cantley, professor of systems biology at Harvard Medical School and Beth Israel Deaconess Medical Center. His team identified PKM2 in a proteomic screen for proteins that preferentially bind to peptides containing phosphotyrosine (pTyr), which is a signature of kinase substrates. Among the usual suspects such as proteins involved in signal transduction, the researchers noticed PKM2, the fetal isoform of a seemingly mundane glycolytic enzyme.

PKM2 has previously been shown to be highly expressed in embryos and tumors but absent in healthy adult cells. ${ }^{6}$ It differs from the adult version of pyruvate kinase by the presence of a 56-aminoacid stretch that binds pTyr.
Cantley's group shows that the pTyr-binding region of PKM2 is required for aerobic glycolysis and tumor growth. Removing this region of PKM2 shifted tumor cell metabolism back to oxidative phosphorylation and decreased tumor size compared with the original tumor size in a mouse model of lung cancer.

He suggested that the presence of fetal PKM2 in addition to the normal adult version may explain why tumors appear to squander their energy through aerobic glycolysis. "You can get 36 [more] molecules of ATP from a molecule of glucose" than cancer cells do, Cantley told SciBX.

More fundamentally, cancers may need PKM2 because tumor cells, like embryos, grow rapidly and require not only energy but also basic biochemical building blocks. By diverting the flow of energy away from mitochondria, cancer cells stockpile the components needed to make new macromolecules through anabolic processes such as gluconeogenesis and lipid synthesis.

"Tumor cells and embryonic cells are busy building up amino acids, nucleic acids, etc., while adult cells are just using glucose for energy," said Cantley. "The difference between these two modes is controlled by PKM2."

\section{No sugar tonight}

PKM2 has previously cropped up as a cancer marker, but its specific role in tumor growth has been mysterious. One company, Thallion Pharmaceuticals Inc., has already brought a PKM2 inhibitor to clinical trials. However, the original proposed mechanism of action of the company's CAP-232 involves a separate role for PKM2 as an alternative receptor for the hormone somatostatin.

CAP-232 is based on findings from György Kéry, professor of medical chemistry at Semmelweis Medical University, whose group synthesized the compound as an inhibitor of somatostatin receptors and later found that it also bound PKM2. CAP-232 promotes nuclear localization of a dimeric form of PKM2, which leads to apoptosis. ${ }^{7}$

"I think this is a beautiful demonstration of the role of PKM2" in glycolysis, said Allan Mandelzys, EVP of licensing at Thallion. Mandelzys called the discovery of a possible link between PKM2 and signal transduction "very exciting."

"The fact that they've demonstrated that pTyr binds to PKM2 is also novel. That's news to us," said Mandelzys, who suggested that regulation of pTyr by kinases might "shift PKM2 from the tetrameric form to the dimeric form" associated with cancer.

CAP-232 is in Phase II trials for renal cell carcinoma and metastatic melanoma.

Mandelzys told SciBX that although further research will be needed to elucidate the mechanism of PKM2's role in cancer, "having additional data validating the target is really nice."

Charles Hart, director of biology at Threshold Pharmaceuticals Inc., called PKM2 a "gatekeeper of glycolysis" and said the reports were "significant new findings that advance our understanding of the unique metabolism" of cancer cells. "Almost all cancer cells have PKM2, which ordinarily should be expressed only in embryos," said Hart. 
Hart agreed with Cantley's interpretation that the abnormal presence of PKM2 in tumors makes the metabolism of cancer cells resemble that of embryos.

Threshold's 2-deoxyglucose (2DG) seeks to exploit the metabolic difference between healthy and cancer cells by clogging up an early step in glycolysis. The glycolytic enzyme hexokinase converts 2DG into 2-deoxyglucose-6-phosphate, which cannot be metabolized any further, causing rapidly dividing cancer cells to run out of gas.

2DG is in Phase I trials to treat solid tumors.

Hart said knocking out other enzymes involved in glycolysis, such as lactate dehydrogenase or pyruvate dehydrogenase kinase (PDK), can also reduce tumor growth. For example, the generic PDK inhibitor dichloroacetate (DCA) reverses the Warburg effect, promotes apoptosis and reduces tumor growth. ${ }^{8}$
The University of Alberta is running a Phase II study of DCA for anaplastic astrocytoma and glioblastoma.

Hart told SciBX that targeting PKM2 together with other glycolytic enzymes could create a powerful combination therapy to starve cancers of sugar. "Whether you do this through PKM2 or through other antiglycolytic strategies, you have a complementary means to a similar end, which is targeting tumor-specific metabolism," he said.

However, Hart cautioned that glycolytic enzymes such as PKM2 can often wear multiple hats. It is not yet known whether PKM2 has other roles unrelated to its enzymatic activity.

"Sometimes things evolve other purposes. Hexokinase is also part of the apoptotic machinery, and glyceraldehyde 3-phosphate dehydrogenase is a transcription factor. So is enolase," said Hart, alluding to newly discovered secondary roles for enzymes originally discovered in glycolysis.

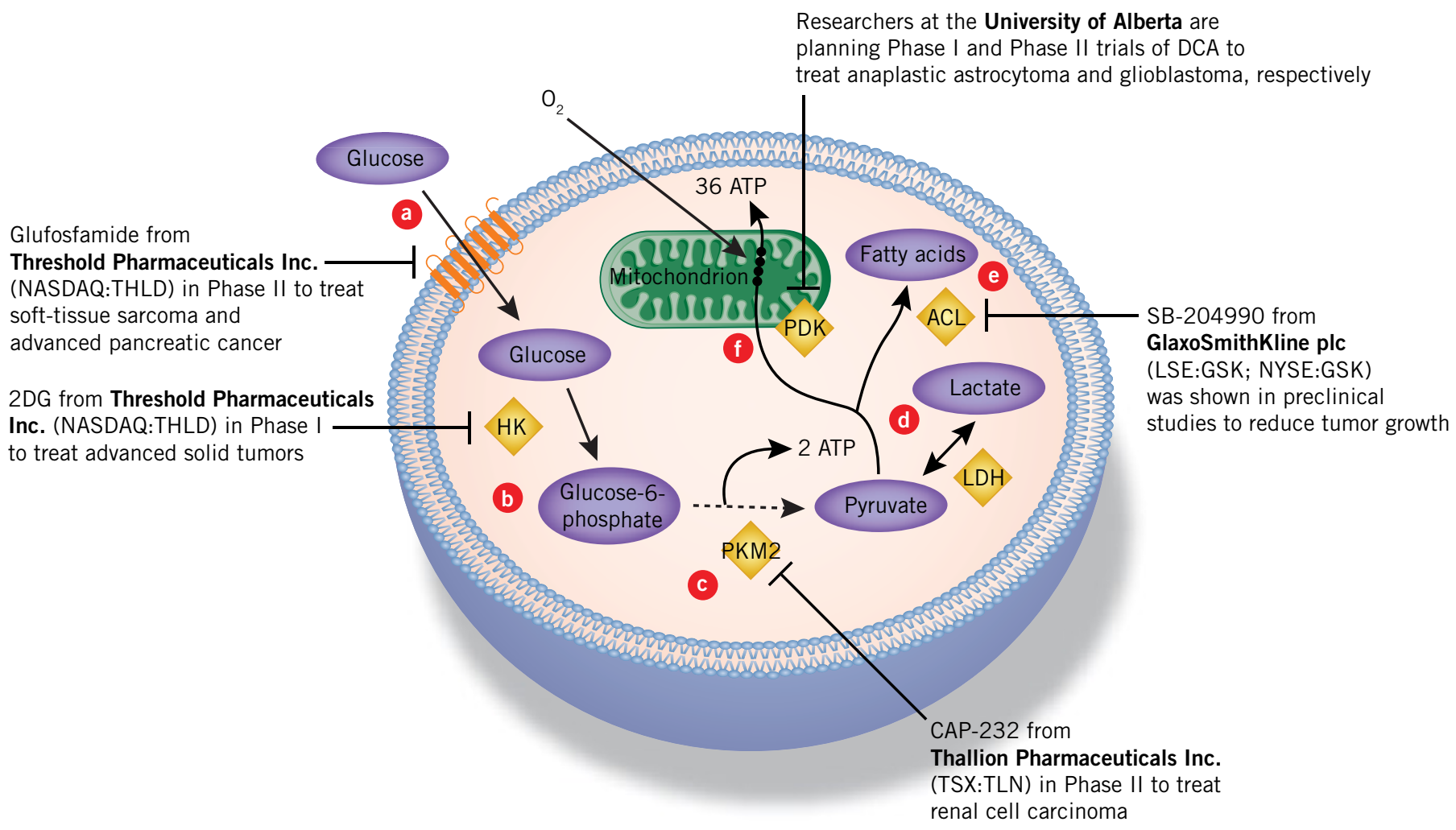

Figure 1. Steps in glycolysis and respiration targeted by cancer therapeutics. Several companies are developing compounds that target steps in glycolysis and related metabolic processes that cancer cells rely upon.

[a] Glufosfamide competes with glucose for transport into the cell. Once inside, glufosfamide turns into a toxic alkylating agent.

[b] Glucose breakdown by hexokinase (HK) is competitively inhibited by 2-deoxyglucose (2DG), which is catabolized by HK into a deadend metabolite that blocks glycolysis.

[c] CAP-232 directs cancer-associated pyruvate kinase isoform M2 (PKM2) toward the nucleus, triggering apoptosis. Agios Pharmaceuticals Inc. is also researching compounds that target PKM2.

[d] Researchers have found that knockdown of lactate dehydrogenase (LDH) with small hairpin RNA decreases glycolysis and cancer cell growth. ${ }^{4}$

[e] ATP citrate lyase (ACL) is inhibited by SB-204990. Preclinical studies have found that treatment with this compound blocks fatty acid synthesis and cancer cell growth. ${ }^{5}$

[f] Inhibition of mitochondrial pyruvate dehydrogenase kinase (PDK), a negative regulator of respiration, by the generic compound dichloroacetate (DCA) lowers tumor glycolysis and promotes apoptosis. Researchers at the University of Alberta are planning trials of DCA. 


\section{TARGETS \& MECHANISMS}

\section{Carb control}

With PKM2 on the map, researchers can now probe how signaling pathways implicated in cancer can regulate glycolysis. The first place to look for signs of signaling pathways that regulate PKM2 would be its pTyr-binding domain. The problem is that the pTyr-containing protein(s) that control PKM2 activity are still unknown, as are the kinases that phosphorylate these proteins. PKM2's binding and regulatory partners will be the focus of future work, Cantley told SciBX.

According to Cantley, likely candidates for interaction with PKM2's pTyr-binding region include the upstream glycolytic enzyme enolase or a downstream enzyme such as lactate dehydrogenase. A close association between PKM2 and either of these enzymes could divert pyruvate away from the mitochondria, promoting anabolic metabolism.

Another potential next step would be to develop therapeutics that specifically target PKM2's unique features, such as the pTyr-binding pocket or an additional site that binds an upstream metabolite, fructose 1,6-bisphosphate.

Cantley thinks the ideal PKM2 therapeutic would shift the enzyme's activity into a more adult-like state, thus putting cancer growth back into low gear without harming normal cells. Slowing down tumor growth in this fashion could boost apoptosis or make it easier for other therapeutics to kill cancer cells. ${ }^{9}$

A patent on the technology described in the papers has been filed by Beth Israel Deaconess Medical Center. Cantley and his collaborators have founded a company, Agios Pharmaceuticals Inc., to identify other metabolic targets in cancer and other cell growth-related diseases, and have licensed the screening platform used to discover PKM2.

According to Cantley, Agios has already identified "proof-of-principle compounds" that target PKM2.

\section{REFERENCES}

1. Christofk, H.R. et al. Nature; published online March 12, 2008; doi:10.1038/ nature06734

Contact: Lewis C. Cantley, Beth Israel Deaconess Medical Center, Boston, Mass. e-mail: Icantely@hms.harvard.edu

2. Christofk, H.R. et al. Nature; published online March 12, 2008; doi:10.1038/nature06667

Contact: Lewis C. Cantley, Beth Israel Deaconess Medical Center, Boston, Mass.

e-mail: Icantely@hms.harvard.edu

3. Gatenby, R.A. \& Gillies, R.J. Nat. Rev. Cancer 4, 891-899 (2004)

4. Fantin, V.R. et al. Cancer Cell 9, 425-434 (2006)

5. Hatzivassiliou, G. et al. Cancer Cell 8, 311-321 (2005)

6. Oremek, G.M. et al. Anticancer Res. 19, 2599-2601 (1999)

7. Stetak, A. et al. Cancer Res. 67, 1602-1608 (2007)

8. Bonnet, S. et al. Cancer Cell 11, 37-51 (2007)

9. Pelicano, H. et al. Oncogene 25, 4633-4646 (2006)

\section{COMPANIES AND INSTITUTIONS MENTIONED}

Agios Pharmaceuticals Inc., Cambridge, Mass.

Beth Israel Deaconess Medical Center, Boston, Mass.

Harvard Medical School, Boston, Mass.

Semmelweis Medical University, Budapest, Hungary

Thallion Pharmaceuticals Inc. (TSX:TLN), Montreal, Quebec, Canada Threshold Pharmaceuticals Inc. (NASDAQ:THLD), Redwood City, Calif. University of Alberta, Edmonton, Alberta, Canada 